\title{
VERTICAL JUMPING PERFORMANCE RELATES TO SPRINTING PERFORMANCE OVER SHORT DISTANCES AND DIFFERENT SECTIONS
}

OPEN ACCESS

Submitted: 21 May 2021 Revised: 27 October 2021 Accepted: 22 November 2021

ORCID iD

Sebastian Möck https://orcid.org/0000-0001-6277-2696 Cite this article as:

Möck, S., Hartmann, R., Wirth, K. (2021).

Vertical jumping performance relates to sprinting performance over short distances and different sections Journal of Applied Sports Sciences, Vol.2, pp. 17-27. DOI: $10.37393 / J A S S .2021 .02 .2$

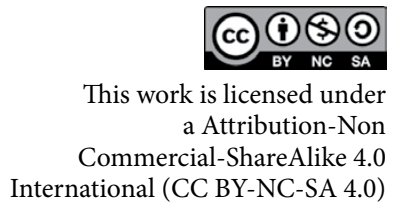

This work is licensed under a Attribution-Non

Commercial-ShareAlike 4.0 International (CC BY-NC-SA 4.0)

\section{Sebastian Möck ${ }^{1}$, René Hartmann ${ }^{2}$, Klaus Wirth ${ }^{3}$}

${ }^{1}$ Department of Exercise Science, Olympic Training and Testing Center of Hessen, Frankfurt am Main, Germany

${ }^{2}$ Institute of Sports Sciences, Johann Wolfgang Goethe-University, Frankfurt am Main, Germany

${ }^{3}$ Sport and Exercise Sciences, University of Applied Sciences

Wiener Neustadt, Austria

\section{ABSTRACT}

A high level of sprinting performance is relevant in various sports. Because of the transition of movement patterns in different sprint sections there is a shift in the relevance of speed-strength of the knee and hip extensors, and stretch-shortening cycle performance seems conceivable. Fifty-six physical education students $(23.70 \pm 3.00$ years, $176.9 \pm 8.10 \mathrm{~cm}, 74.20 \pm 10.30 \mathrm{~kg})$ were investigated. They performed sprints up to $30 \mathrm{~m}$ in which different sections were analyzed and vertical jumps (squat jump, countermovement jump, drop jump from different dropping heights). Vertical jumping tests in squat jump and countermovement jump revealed mean values of $31.95 \pm 6.56 \mathrm{~cm}$ and $34.28 \pm 7.47 \mathrm{~cm}$, respectively, while the drop jumps showed mean RSI values between $155.11 \pm 36.77$ and $168.24 \pm 36.29$ dependent on the dropping height. The sprint test showed a mean performance of $4.464 \pm .343 \mathrm{~s}(30 \mathrm{~m})$. The correlational analysis showed significant correlations $(p<.01)$ for vertical jumping height with all sprinting sections $(r=-.652$ to -.834$)$. Drop jump performance also showed significant correlations $(p<.01)$ with all the sections $(r=-.379$ to -.594$)$.

The results let us hypothesize that the observed sample generated similar ground-reaction forces in the sprint and drop jump from a height of $40 \mathrm{~cm}$.

Keywords: Stretch-Shortening Cycle, Movement Speed, Speed-Strength

\section{INTRODUCTION}

A high level of performance in the sprint is not only important for sprinters in track and field but also for athletes in jumping and throwing events as well as in team sports (Brechue, Mayhew, Piper, 2010; Chelly et al., 2010; Comfort, Bullock, Pearson, 2012; Di Salvo et al., 2010).

In team sports, sprints over distances of 5-20m (Abdelkrim et al., 2010, Brechue et al., 2010; Chelly et al., 2010; Comfort et al., 2012; Di Salvo et al., 2010) or durations of $1.8-2.1 \mathrm{~s}$ (Abdelkrim, El Fazaa, El Ati, 2007; Spencer et al., 2004) are common and may be character- ized as short events. Therefore, Cronin \& Hansen (2005) emphasized the importance of the ability to produce a fast acceleration for these athletes.

In track and field, the sprint is commonly divided into phases of positive acceleration, maximum velocity, and negative acceleration (Mann, Herman, 1985; Seagrave, 1996; Volkov, Lapin, 1979). The technical recommendations for sprinting assume that in the initial acceleration phase, because of the stronger forward lean, the drive is predominantly produced by the knee extensor muscles and subsequently, 
after gradually raising the upper body, shifts towards hamstring musculature (Vonstein, 1996). Substantial forward lean can also be observed in sprints from a standing starting position (Frost \& Cronin, 2011). Scientific studies support this claim (Simonsen, Thomsen, Klausen, 1985, Wiemann, Tidow, 1995). Therefore, the knee extensor musculature's task during the maximal velocity phase is primarily to counteract a lowering of the body's center of mass (Simonsen et al., 1985, Wiemann, Tidow, 1995). Thus, the stiffness of the knee joint seems to be a better indicator than the maximal dynamic force of the extensors for this phase (Bret, Rahmani, Dufour, Messonnier, Lacour, 2002; Chelly, Denis, 2001). Furthermore, the ability to produce as much horizontal force as possible during the ground contact seems to be of more importance than the absolute amount of force produced (Morin, Edouard, Samozino, 2011; Morin et al., 2012). Because of these circumstances and the reduction of ground contact times throughout the sprint (Mero, Komi, Gregor, 1992), which cause shorter periods of time to transfer force to the ground, the relevance of an athlete's maximum strength seems to decrease with increasing distance while the rate of force development gains importance (Zatsiorsky, 2003, Zatsiorsky, Kraemer, 2006).

Vertical jumps like the squat jump (SJ) and the countermovement jump (CMJ) are regarded as standard tests for the speed-strength performance of the lower extremities (Bret et al., 2002, Hartmann et al., 2012, Kukolj, Ropret, Ugarkovic, Jaric, 1999, Loturco et al., 2015, Wirth et al., 2016). Previous research observed significant relationships between vertical jumping performance and sprinting speed, al- though contradictory findings exist. The study of Loturco et al. (2015), for example, showed significant correlations for SJ and CMJ with sprinting performance after 10,30 , and $50 \mathrm{~m}$ for competitive sprinters $0.756 \leq r \geq 0.857$ ), whereas Kukolj et al. (1999) did not observe significant correlations for sports students after $15 \mathrm{~m}$ at all and only moderate correlations of $r=.48$ after $30 \mathrm{~m}$.

The impact of the gradual change in posture due to the transition from the acceleration phase to the maximal velocity phase on the relevance of the strength-speed performance of knee and hip extensors for sprinting performance remains unclear.

Because of the short ground contact times of $0.08-0.11 \mathrm{~ms}$ with little change in knee angle (Mero et al., 1992), a great performance capacity in the fast stretch-shortening cycle (SSC) with a duration $<200 \mathrm{~ms}$ (Horita, Komi, Nicol, Kyröläinen, 1996, Horita, Komi, Nicol, Kyröläinen, 2002) is regarded as a necessity for maximizing sprinting velocity (Mero et al., 1992). Nevertheless, empirical findings utilizing the vertical drop jump (DJ) are rather scarce and show contradictory results (Cronin, Hansen 2005, Cunha et al., 2007, Hennessy, Kilty, 2001). It is noteworthy that these studies only used a single dropping height to determine SSC performance. This procedure neglects the fact that the reactive strength is linked to the dropping height, and an individual optimum exists because of the differences in the stretch velocity that elicits the reflex mechanisms upon ground contact (Komi, 2003, Schmidtbleicher, 1992).

Here as well, the relevance of DJ performance throughout the different sprinting pha- 
ses remains unclear to date.

Therefore, we hypothesize that running distance increases the relationship between sprint and jumping performance.

\section{MATERIAL \& METHODS}

This study aims to investigate the relationships between different vertical jumps and sprinting performance over short distances and different sections. For this purpose, 56 physical education students participated in this investigation. Concentric jump performance was measured by SJ, slow-reactive by CMJ. As a test of the fast-reactive movement behavior with different intensities, the DJ with impulse duration of less than $200 \mathrm{~ms}$ was used. The sprint performance was measured over $30 \mathrm{~m}$, in which times were taken by double-photoelectric barriers after $5,10,15,20,25$, and $30 \mathrm{~m}$, respectively. The measurements were performed on three different days with a week in-between each test and included a familiarization session and a testing session for both tests.

The following parameters were determined: Jumping height in the SJ and CMJ and reactive strength index (RSI) in the DJ from different heights as well as the spiriting times $0-5 \mathrm{~m}$, $0-10 \mathrm{~m}, 0-15 \mathrm{~m}, 0-20 \mathrm{~m}, 0-25 \mathrm{~m}, 0-30 \mathrm{~m}$ and 5-10m, 10-15m, 15-20m, 20-25m, 25-30m.

\section{Subjects}

Fifty-six physical education students (40m, 16f) with a mixed sports background and activity levels participated in this investigation. The sporting backgrounds were various sport games $(\mathrm{N}=25)$, metric sports (track and field, swimming; $\mathrm{N}=15)$, compositional sports such as gymnastics or dancing $(\mathrm{N}=6)$ and combat sports $(\mathrm{N}=10)$. The mean age was $23.7 \pm 3.0$ years, mean height $176.9 \pm 8.1 \mathrm{~cm}$ and weight $74.2 \pm 10.3 \mathrm{~kg}$. Each subject was informed of the experimental risks involved with the research. All subjects provided informed written consent. The research design was approved by the institutional review board. The study was carried out with respect to the use of human subjects and according to the Declaration of Helsinki.

\section{Sprint test}

The sprint test was performed after an individual warm-up of 10 minutes over a distance of $30 \mathrm{~m}$. This distance was chosen because it displays a relevant motor task in many sports such as soccer, football, rugby or field hockey (Brechue et al., 2010, Chelly et al., 2010, Comfort et al., 2012). Five attempts were measured with a rest period of 5 minutes between attempts. For time tracking a double-photoelectric barrier system by Refitronic (Schmitten, Germany) was used, whose measurement error is $<0,1 \%$ according to the manufacturer. The running times were detected after 5, 10, $15,20,25$, and $30 \mathrm{~m}$, respectively.

The start was executed in an upright position $50 \mathrm{~cm}$ in front of the first light gate. No command was given, and every tested individual started the test at an individually chosen point of time. Test-retest reliability of $r=.94$ - .98 $(p<.05)$ is indicated for this test (Keiner, Sander, Wirth, Hartmann, Yaghobi, 2014).

\section{Jump tests}

The detection of jump heights and contact times of the jumps described below was carried out using a contact mat measuring system 
(Refitronic, Schmitten, Germany) with an error in the time measurement $<0.15$ according to the manufacturer. In the case of SJ and CMJ, 5 attempts per jump were carried out with an inter jump rest period of at least $30 \mathrm{~s}$. In the case of the DJ, 5 attempts were made for each height with the same intra- and inter-serial pause duration.

\section{Squat Jump}

The SJ was performed from a static starting position with knee flexion of about $90^{\circ}$ with hands fixed at the hips and the upper body as upright as possible. Countermovements of any kind were not permitted. Wirth et al. (2016) state test-retest reliability of ICC $=.88$ for this test.

\section{Countermovement Jump}

To detect the slowly-reactively reached jump height, the CMJ was carried out by utilizing a countermovement from an upright starting position to a knee flexion of about $90^{\circ}$ with hands fixed at the hips while keeping the upper body as upright as possible. This prevents support from swinging the arms or extreme torso propulsion. Wirth et al. (2016) state test-retest reliability of $\mathrm{ICC}=.93$ for this test.

\section{Drop Jump}

The DJ was used to assess the fast-reactive strength. The tested drop heights were 16, 24, 32,40 , and $48 \mathrm{~cm}$, respectively. The subject stood upright on a box with hands fixed on the hips in the starting position. From this position, the subject started with a forward swing of a leg from the box and aimed to jump as high as possible after a short contact with the ground, paying attention to a bouncing jump execution. Ground contact times of $\geq 200 \mathrm{~ms}$, disengagement of the hands from the hips, ground contact of the heels during the takeoff, or excessive joint angle enlargement in the knee and hip joint, led to an invalid jump. The reactive strength capability is represented by the reactive strength index (RSI). This was calculated according to Wirth, Sander, Keiner, Schmidtbleicher (2011) from the ground contact time and the jump height using the formula: RSI = jump height in $\mathrm{mm}$ / contact time in ms x 100. The test-retest reliability for the RSI from varying drop heights is ICC $=.87-.90$ (Wirth et al., 2016) and is therefore classified as very high. Subjects were instructed to achieve a maximum jump height with minimal ground contact time. An exclusive request for maximum jump height may result in jump characteristics of the CMJ (Young, Pryor, Wilson, 1995), which is an acquisition of performance in the slow SSC and, therefore, would not be appropriate.

\section{Statistical analysis}

The data was analyzed with the use of SPSS 11.5 (SPSS, Inc., Chicago, IL, USA). Kolmogorov-Smirnov test was used to check for normal distribution. As this test revealed no significant result, Pearson's product-moment correlation was used to determine the strength of the relationships. The level of significance for all tests was set a priori to $p \leq .05$.

According to Keiner et al. (2014) the correlation coefficients were classified as follows: $0=$ no correlation, $0<|r|<.2=$ very weak correlation, $.2<|r|<.4=$ weak correlation, .4 $<|r|<.6=$ medium correlation, $.6<|r|<.8=$ strong correlation, $.8<|r|<1.0=$ very strong 
correlation, 1 = perfect correlation.

Additionally, for CMJ and the DJ RSI with the greatest correlation to sprinting performance, the fastest and slowest fifty percent of the participants based on $30 \mathrm{~m}$ sprint performance were compared via a two-tailed independent samples $t$-Test and Cohen's $d$. The effect sizes were classified according to Cohen (1988): $d>.2=$ small effect, $d>.5=$ medium effect, $d>.8=$ large effect.

\section{RESULTS}

The descriptive statistics are displayed in Table 1. For the sprinting sections, mean in- terval times between $0.768 \pm 0.054 \mathrm{~s}$ and 0.628 $\pm 0.062 \mathrm{~s}$ were obtained, while the cumulative sprinting times showed means between 4.464 $\pm 0.343 \mathrm{~s}$ and $1.095 \pm 0.071 \mathrm{~s}$. The jumping tests revealed mean jumping heights of 31.95 $\pm 6.56 \mathrm{~cm}$ in the $\mathrm{SJ}$ and $34.28 \pm 7.47 \mathrm{~cm}$ in the CJ, while the RSI in the drop jumps showed mean values between $155.11 \pm 36.77$ and $168.24 \pm 36.29$. Mean DJ RSI increased with increasing dropping height up to $32 \mathrm{~cm}$ and showed a slight decline with further increases up to $48 \mathrm{~cm}$. The sample showed coefficients of variation between $6.44 \%$ and $7,68 \%$ for the sprinting times.

Table 1. Descriptive data of the conducted sprint and jump tests

\begin{tabular}{lcccc}
\hline & Mean & SD & Min & Max \\
\hline T 5m [s] & 1.095 & 0.071 & 0.947 & 1.256 \\
T 10m [s] & 1.863 & 0.120 & 1.661 & 2.139 \\
T 15m [s] & 2.546 & 0.173 & 2.258 & 2.955 \\
T 20m [s] & 3.201 & 0.228 & 2.822 & 3.737 \\
T 25m [s] & 3.836 & 0.288 & 3.373 & 4.509 \\
T 30m [s] & 4.464 & 0.343 & 3.910 & 5.271 \\
T 5-10m [s] & 0.768 & 0.054 & 0.680 & 0.883 \\
T 10-15m [s] & 0.683 & 0.055 & 0.593 & 0.816 \\
T 15-20m [s] & 0.654 & 0.056 & 0.564 & 0.782 \\
T 20-25m [s] & 0.639 & 0.059 & 0.551 & 0.772 \\
T 25-30m [s] & 0.628 & 0.062 & 0.537 & 0.762 \\
SJ [cm] & 31.95 & 6.56 & 17.60 & 49.30 \\
CMJ [cm] & 34.28 & 7.47 & 17.60 & 50.20 \\
RSI 16 & 155.11 & 36.77 & 87.00 & 244.00 \\
RSI 24 & 162.69 & 35.99 & 97.00 & 245.00 \\
RSI 32 & 168.24 & 36.29 & 101.00 & 235.00 \\
RSI 40 & 166.80 & 38.95 & 91.00 & 253.00 \\
RSI 48 & 163.00 & 38.95 & 74.00 & 256.00 \\
\hline
\end{tabular}

The calculation of the correlations between sprinting performance and jumping heights in the SJ and the CMJ revealed significant, strong correlations $(p \leq .01)$ for all parameters, with a tendency for larger coefficients with increasing running distance (Table 2). 
Table 2. Correlations of sprint performance with jumping height in SJ and CMJ

\begin{tabular}{lll}
\hline & SJ & CMJ \\
\hline T $5 \mathrm{~m}$ & $-.671^{*}$ & $-.693^{*}$ \\
T $10 \mathrm{~m}$ & $-.722^{*}$ & $-.746^{*}$ \\
T $15 \mathrm{~m}$ & $-.741^{*}$ & $-.766^{*}$ \\
T $20 \mathrm{~m}$ & $-.658^{*}$ & $-.652^{*}$ \\
T $25 \mathrm{~m}$ & $-.763^{*}$ & $-.813^{*}$ \\
T $30 \mathrm{~m}$ & $-.763^{*}$ & $-.793^{*}$ \\
T $5-10 \mathrm{~m}$ & $-.723^{*}$ & $-.747^{*}$ \\
T $10-15 \mathrm{~m}$ & $-.756^{*}$ & $-.783^{*}$ \\
T $15-20 \mathrm{~m}$ & $-.769^{*}$ & $-.812^{*}$ \\
T $20-25 \mathrm{~m}$ & $-.757^{*}$ & $-.805^{*}$ \\
T $25-30 \mathrm{~m}$ & $-.790^{*}$ & $-.834^{*}$ \\
\hline
\end{tabular}

Note: ${ }^{\star}=p<.01$

The calculation of the correlations between larger coefficients with increasing drop height sprinting performance and RSI in the DJ re- in the DJ (Table 3). The greatest correlation covealed significant, medium correlations ( $p \leq$ efficients were obtained for a dropping height $.01)$ for all parameters, with a tendency for of $40 \mathrm{~cm}$.

Table 3. Correlations of sprint performance with the RSI in the DJ

\begin{tabular}{llllll}
\hline & RSI 16 & RSI 24 & RSI 32 & RSI 40 & RSI 48 \\
\hline T 5m & $-.379^{*}$ & $-.441^{*}$ & $-.454^{*}$ & $-.498^{*}$ & $-.403^{*}$ \\
T 10m & $-.412^{*}$ & $-.458^{*}$ & $-.504^{*}$ & $-.533^{*}$ & $-.437^{*}$ \\
T 15m & $-.424^{*}$ & $-.471^{*}$ & $-.524^{*}$ & $-.554^{*}$ & $-.458^{*}$ \\
T 20m & $-.425^{*}$ & $-.478^{*}$ & $-.542^{*}$ & $-.569^{*}$ & $-.476^{*}$ \\
T 25m & $-.418^{*}$ & $-.472^{*}$ & $-.530^{*}$ & $-.559^{*}$ & $-.449^{*}$ \\
T 30m & $-.431^{*}$ & $-.489^{*}$ & $-.561^{*}$ & $-.586^{*}$ & $-.497^{*}$ \\
T 5-10m & $-.415^{*}$ & $-.438^{*}$ & $-.521^{*}$ & $-.528^{*}$ & $-.441^{*}$ \\
T 10-15m & $-.434^{*}$ & $-.480^{*}$ & $-.550^{*}$ & $-.581^{*}$ & $-.485^{*}$ \\
T 15-20m & $-.410^{*}$ & $-.478^{*}$ & $-.572^{*}$ & $-.594^{*}$ & $-.519^{*}$ \\
T 20-25m & $-.413^{*}$ & $-.490^{*}$ & $-.566^{*}$ & $-.588^{*}$ & $-.484^{*}$ \\
T 25-30m & $-.434^{*}$ & $-.488^{*}$ & $-.552^{*}$ & $-.566^{*}$ & $-.468^{*}$ \\
\hline
\end{tabular}

The comparison of the fastest and slowest the faster subjects showing statistically higher $50 \%$ of the subjects revealed significant differ- jumping performance (Table 4). ences between the groups in both jumps, with 
Table 4. Comparison of the fastest and slowest participants.

\begin{tabular}{llllc}
\hline & Fastest 50\% & Slowest 50\% & p & d \\
\hline CMJ & 38.59 & $29.98^{*}$ & .000001 & 1.40 \\
RSI 40 & 180.93 & $155.48^{*}$ & .006 & .71 \\
\hline
\end{tabular}

Note: $*$ = significant group difference

\section{DISCUSSION}

The results from the present study showed consistently significant relationships $(p<.01)$ between vertical jumping performance and sprinting performance up to $30 \mathrm{~m}$ for the observed sample and an increase in the relationships with running distance.

The jumping heights in SJ and CMJ showed strong significant correlations for all sections. The values of the obtained coefficients were slightly lower than the ones obtained by Loturco et al. (2015) with competitive sprinters but clearly greater than the results of Kukolj et al. (1999), who also examined physical education students. One approach to explain the different results with similar participants might be the hypothesis that the participants of this study might have had lower differences regarding the movement pattern in the sprint so that the capacity to apply force on the ground gained relevance for the reached sprinting velocity.

Regarding the correlations of the RSI in the DJ with the different sprint section significant, medium coefficients $p<.01$ ) could be observed. The greatest correlations were obtained for a dropping height of $40 \mathrm{~cm}$. The obtained relationships are in accordance with those of Cunha et al. (2007), who measured a coefficient of $r=-.684$ between sprint performance and RSI with a dropping height of $24 \mathrm{~cm}$ using a collective of participants with mixed performance level. Using a dropping height of $30 \mathrm{~cm}$,
Hennessy \& Kilty (2001) obtained an even greater relationship of $r=-.79(p<0.05)$ with sprint performance up to $30 \mathrm{~m}$ in active sprinters. Limiting the value of these comparisons, it is to note that the mentioned studies only used one dropping height to determine SSC performance. Since SSC performance shows a development towards an optimum when altering the dropping height (Komi, 2003, Schmidtbleicher, 1992), the performance of the observed collectives might have easily been over-or underestimated by Cunha et a. (2007) and Hennessy \& Kilty (2001).

With increasing sprinting distance, an increase in the correlation coefficients with the RSI can be observed. In this regard, the increasing ground reaction forces with simultaneously decreasing ground contact times (Mann, Herman, 1985) are to be considered. This trend should let an increasing stretch velocity with the associated reflex mechanisms be expected. Additionally, the results by Ishikawa \& Komi (2007) indicated a shift of the achilles tendon reflex's mechanical effect from the breaking phase to the propulsive phase of the ground contact with increasing running speed.

The comparison of the different dropping heights in the DJ showed that the greatest correlations were obtained using a height of $40 \mathrm{~cm}$. This might reflect the mean proficiency level of the sample and could reflect the optimum dropping height for the studied sample. 
If the dropping height exceeds the individuals' optimum height, subtle changes regarding the movement pattern can be observed that manifest in greater joint movements of the knee and hip joints (Bobbert, 1990, Bobbert, Huijing, Van Ingen Schenau, 1987). This pattern serves to reduce the ground reaction forces that probably would exceed the individuals' capacity without altering the movement pattern. This hypothesis is emphasized by the observations of Bobbert et al. (1987), who obtained similar joint moments in the knee and hip joints for DJ with a dropping height of $20 \mathrm{~cm}$ and $40 \mathrm{~cm}$, respectively, whereas the impulse and the vertical component of the ground reaction forces in the push-off rose with increasing dropping height. A further increase of the dropping height to 60 $\mathrm{cm}$ resulted in a decrease of all the mentioned parameters. Additionally, the authors stated that the participants were not able to prevent their heels from touching the ground anymore, which further underlines the conclusion that this dropping height exceeded the participants' capacity. Although these results of Bobbert et al. (1987) are limited in their power because the ground reaction times exceeded $200 \mathrm{~ms}$, which is why a fast SSC cannot be expected (Horita et al., 1996, Horita et al., 2002), they clearly provide evidence for the changes of the ground reaction forces with modified jumping patterns.

Considering the importance of the ability to produce a high amount of force in the horizontal plane for high sprint velocities (Morin et al., 2011, Morin et al., 2012), one might argue that DJ in a vertical motion does not reflect the necessary movement pattern. But despite the observation that horizontal force production is indeed highly important for sprint performance, the importance of vertical DJ performance still seems eligible. Since propulsion in the sprint from about $20 \mathrm{~m}$ in the run is believed to be realized primarily via hip extension with great swing velocity (Bezodis, Kerwin, Salo, 2008, Hunter, Marshall, McNair, 2005, Seagrave, 1996), the main contribution of knee and ankle joints is the amortization of the ground reaction forces' vertical component (Hunter, Marshall, McNair, 2004, Hunter et al., 2005). To provide for this task while avoiding to high an amount of horizontal breaking forces, ideally, the knee and ankle joints are positioned nearly vertically stacked when contacting the ground slightly in front of the vertical projection of the center of mass (Hunter et al., 2004, Hunter et al., 2005).

The results of the correlational analysis are further supported by the exemplary comparisons of the fastest and slowest 50\% in CMJ performance and DJ RSI from a dropping height of $40 \mathrm{~cm}$. Significant group differences could be observed in both cases, which underlines the need of a high SSC capacity for sprint performance.

\section{CONCLUSION}

The results of the present study indicate medium to strong correlations between vertical jumping and sprinting performance with a tendency to increasing relationships with increasing sprinting distance. For the investigated sample, the greatest relationships with DJ performance could be observed with a dropping height of $40 \mathrm{~cm}$. Whether this observation can be possibly underlined by the ground reaction forces in these two movements tasks, needs to be addressed by additional research. 


\section{REFERENCES}

Abdelkrim, N. B., El Fazaa, S., \& El Ati, J. (2007). Time-motion analysis and physiological data of elite under-19-year-old basketball players during competition, British Journal of Sports Medicine, 41(2), pp. 69-75.

Abdelkrim, N. B., Castagna, C., Jabri, I., Battikh, T., El Fazaa, S., \& El Ati, J. (2010). Activity profile and physiological requirements of junior elite basketball players in relation to aerobic-anaerobic fitness, The Journal of Strength \& Conditioning Research, 24(9), pp. 2330-2342.

Bezodis, I. N., Kerwin, D. G., \& Salo, A. I. (2008). Lower-limb mechanics during the support phase of maximum-velocity sprint running, Medicine and Science in Sports and Exercise, 40(4), pp. 707-715.

Bobbert, M. F. (1990). Drop jumping as a training method for jumping ability, Sports Medicine, 9(1), pp. 7-22.

Bobbert, M. F., Huijing, P. A., \& Van Ingen Schenau, G. J. (1987). Drop jumping. II. The influence of dropping height on the biomechanics of drop jumping, Medicine and Science in Sports and Exercise, 19(4), 339-346.

Brechue, W. F., Mayhew, J. L., \& Piper, F. C. (2010). Characteristics of sprint performance in college football players, The Journal of Strength \& Conditioning Research, 24(5), pp. 1169-1178.

Bret, C., Rahmani, A., Dufour, A. B., Messonnier, L., \& Lacour, J. R. (2002). Leg strength and stiffness as ability factors in 100 $\mathrm{m}$ sprint running, The Journal of Sports Medicine and Physical Fitness, 42(3), pp. 274-281.

Chelly, M. S., Chérif, N., Amar, M. B., Hermassi, S., Fathloun, M., Bouhlel, E., ... \& She- phard, R. J. (2010). Relationships of peak leg power, 1 maximal repetition half back squat, and leg muscle volume to 5-m sprint performance of junior soccer players, The Journal of Strength \& Conditioning Research, 24(1), pp. 266-271.

Chelly, S. M., \& Denis, C. (2001). Leg power and hopping stiffness: relationship with sprint running performance, Medicine and Science in Sports and Exercise, 33(2), pp. 326333.

Cohen, J. (1988). Statistical Power Analysis for the Behavioral Sciences. Hillsdale, NJ: Lawrence Erlbaum Associates.

Comfort, P., Bullock, N., \& Pearson, S. J. (2012). A comparison of maximal squat strength and 5-, 10-, and 20-meter sprint times, in athletes and recreationally trained men, The Journal of Strength \& Conditioning Research, 26(4), pp. 937-940.

Cronin, J. B., \& Hansen, K. T. (2005). Strength and power predictors of sports speed, The Journal of Strength \& Conditioning Research, 19(2), pp. 349-357.

Cunha, L., Ribeiro, J., Fernandes, O., Valamatos, M., Pinto, R., \& Santos, P. (2007). The relationships between sprint run and strength parameters in young athletes and non-athletes. In H. J. Menzel, \& M. H. Chagas (Eds), Proceedings of the 25th International Symposium on Biomechanics in Sports, Konstanz: International Society of Biomechanics in Sports, pp. 319-322.

Di Salvo, V., Baron, R., González-Haro, C., Gormasz, C., Pigozzi, F., \& Bachl, N. (2010). Sprinting analysis of elite soccer players during European Champions League and UEFA Cup matches, Journal of Sports Scienc- 
es, 28(14), pp. 1489-1494.

Frost, D. M., \& Cronin, J. B. (2011). Stepping back to improve sprint performance: a kinetic analysis of the first step forwards, The Journal of Strength \& Conditioning Research, 25(10), pp. 2721-2728.

Hartmann, H., Wirth, K., Klusemann, M., Dalic, J., Matuschek, C., \& Schmidtbleicher, D. (2012). Influence of squatting depth on jumping performance, The Journal of Strength \& Conditioning Research, 26(12), pp. 32433261.

Hennessy, L., \& Kilty, J. (2001). Relationship of the stretch-shortening cycle to sprint performance in trained female athletes, The Journal of Strength \& Conditioning Research, 15(3), pp. 326-331.

Horita, T., Komi, P. V., Nicol, C., \& Kyröläinen, H. (1996). Stretch shortening cycle fatigue: interactions among joint stiness, reflex, and muscle mechanical performance in the drop jump, European Journal of Applied Physiology and Occupational Physiology, 73(5), pp. 393-403.

Horita, T., Komi, P., Nicol, C., \& Kyröläinen, H. (2002). Interaction between pre-landing activities and stiffness regulation of the knee joint musculoskeletal system in the drop jump: implications to performance, European Journal of Applied Physiology, 88(1-2), pp. 76-84.

Hunter, J. P., Marshall, R. N., \& McNair, P. J. (2004). Interaction of step length and step rate during sprint running, Medicine and Science in Sports and Exercise, 36(2), pp. 261271.

Hunter, J. P., Marshall, R. N., \& McNair, P. J. (2005). Relationships between ground reaction force impulse and kinematics of sprint-running acceleration, Journal of Applied Biomechanics, 21(1), pp. 31-43.

Ishikawa, M., \& Komi, P. V. (2007). The role of the stretch reflex in the gastrocnemius muscle during human locomotion at various speeds, Journal of Applied Physiology, 103(3), pp. 1030-1036.

Keiner, M., Sander, A., Wirth, K., Hartmann, H., \& Yaghobi, D. (2014). Correlations between maximal strength tests at different squat depths and sprint performance in adolescent soccer players, American Journal of Sports Science, 2(1), pp. 1-7.

Komi, P. V. (2003). Stretch-Shortening-Cycle. In P. V. Komi (Ed), Strength and Power in Sport (pp. 184-202). Oxford: Blackwell.

Kukolj, M., Ropret, R., Ugarkovic, D., \& Jaric, S. (1999). Anthropometric, strength, and power predictors of sprinting performance, Journal of Sports Medicine and Physical Fitness, 39(2), pp. 120.

Loturco, I., D’Angelo, R. A., Fernandes, V., Gil, S., Kobal, R., Abad, C. C., ... \& Nakamura, F. Y. (2015). Relationship between sprint ability and loaded/unloaded jump tests in elite sprinters, The Journal of Strength \& Conditioning Research, 29(3), pp. 758-764.

Mann, R., \& Herman, J. (1985). Kinematic analysis of Olympic sprint performance: men's 200 meters, Journal of Applied Biomechanics, 1(2), pp. 151-162.

Mero, A., Komi, P. V., \& Gregor, R. J. (1992). Biomechanics of sprint running, Sports Medicine, 13(6), pp. 376-392.

Morin, J. B., Edouard, P., \& Samozino, P. (2011). Technical ability of force application as a determinant factor of sprint performance, Medicine and Science in Sports and Exercise, 
43(9), pp. 1680-1688.

Morin, J. B., Bourdin, M., Edouard, P., Peyrot, N., Samozino, P., \& Lacour, J. R. (2012). Mechanical determinants of 100-m sprint running performance, European Journal of Applied Physiology, 112(11), pp. 3921-3930.

Schmidtbleicher, D. (1992). Training for power events. In P. V. Komi (Ed), Strength and Power in Sport, Oxford: Blackwell, pp. 381-395.

Seagrave, L. (1996). Introduction to sprinting, New Studies in Athletics, 11, pp. 93-114.

Simonsen, E. B., Thomsen, L., \& Klausen, K. (1985). Activity of mono-and biarticular leg muscles during sprint running, European Journal of Applied Physiology and Occupational Physiology, 54(5), pp. 524-532.

Spencer, M., Lawrence, S., Rechichi, C., Bishop, D., Dawson, B., \& Goodman, C. (2004). Time-motion analysis of elite field hockey, with special reference to repeated-sprint activity, Journal of Sports Sciences, 22(9), pp. 843-850.

Volkov, N. I., \& Lapin, V. I. (1979). Analysis of the velocity curve in sprint running, Medicine and Science in Sports, 11(4), pp. 332.

Vonstein, W. (1996). Some reflections on maximal speed sprinting technique, New Stud- ies in Athletics, 11, pp. 161-165.

Wiemann, K., \& Tidow, G. (1995). Relative activity of hip and knee extensors in sprinting-implications for training, New Studies in Athletics, 10, pp. 29-49.

Wirth, K., Hartmann, H., Sander, A., Mickel, C., Szilvas, E., \& Keiner, M. (2016). The impact of back squat and leg-press exercises on maximal strength and speed-strength parameters, The Journal of Strength \& Conditioning Research, 30(5), pp. 1205-1212.

Wirth, K., Sander, A., Keiner, M., \& Schmidtbleicher, D. (2011). Stretch-Shortening-Cycle Performance of Active and Inactive Children and Adolescents, German Journal of Sports Medicine, 62(11), pp. 345-350.

Young, W. B., Pryor, J. F., \& Wilson, G. J. (1995). Effect of Instructions on characteristics of countermovement and drop jump performance, The Journal of Strength \& Conditioning Research, 9(4), pp. 232-236.

Zatsiorsky, V. M. (2003). Biomechanics of strength and strength training. In P. V. Komi (Ed), Strength and Power in Sport, Oxford: Blackwell, pp. 439-487.

Zatsiorsky, V. M., \& Kraemer, W. J. (2006). Science and practice of strength training. Champaign: Human Kinetics.

\section{Corresponding author:}

Sebastian Möck

Department of Exercise Science, Olympic Training and Testing Center of Hessen, Frankfurt am Main, GERMANY E-mail: smoeck@lsbh.de 